\title{
Stochastic background from extra-galactic double neutron stars
}

\author{
T. Regimbau and B. Chauvineau \\ Dpt. ARTEMIS \\ Observatoire de la Côte d'Azur, \\ BP 42906304 Nice, France \\ E-mail: regimbau@oca.eu; chauvineau@oca.eu
}

\begin{abstract}
.
We present Monte Carlo simulations of the extra galactic population of inspiralling double neutron stars, and estimate its contribution to the astrophysical gravitational wave background, in the frequency range of ground based interferometers, corresponding to the last thousand seconds before the last stable orbit when more than 96 percent of the signal is released. We show that sources at redshift $z>0.5$ contribute to a truly continuous background which may be detected by correlating third generation interferometers.
\end{abstract}

\section{Introduction}

Double neutron stars (DNSs) are very promising sources of gravitational waves for both ground based interferometers, which are sensitive to the last phase of the coalescence and the collapse, and the space detector LISA, which is expected to detect the continuous low frequency inspiral phase. In a previous work, we presented new estimates of the merging rate in the local Universe (including the contribution from ellipticals and taking into account the star formation history derived directly from observation) and discussed its consequences for the first generations of ground based interferometers. We predicted a detection every 148 and 125 years with Virgo and LIGO in their initial configuration, and up to 6 detections per year in their advanced configuration [1, 2]. In addition to the emission from the nearest DNSs, it is expected that the superposition of a large number of unresolved sources produces a stochastic background. The background from the low frequency inspiral phase of various populations of compact binaries, which represents the main source of confusion noise for LISA, was studied intensively in the past decades (see for instance [3, 4, 5, 6, 7] for the galactic foregrounds, and [8, 9, 10, 11] for the extra-galactic contribution). These predictions usually rely on binary evolution codes to estimate initial parameters such as eccentricity, mass ratio, orbital separation, which introduce large uncertainties due to the difficulty modelling mass loss and mass exchanges. In this work, we investigate the stochastic background produced by the extragalactic population of DNSs, carrying on with the the previous estimates of [12, 13]. 
We are interested in the last phase of the coalescence, up to the last stable orbit (LSO), at $\mathrm{kHz}$ frequencies, when the system is in circular orbit and when most of the $\mathrm{GW}$ energy is released. The article will be organized as follow: In section 1, we present a direct calculation of the spectral properties of the stochastic background; in section 2 , we discuss the detection of the background with different generations of terrestrial interferometers; in section 3, we present Monte Carlo simulations of the extra-galactic population of DNSs and its contribution to the GW background; and finally in section 5 , we summarize our results and discuss the interest of the Monte Carlo simulations for source modelling and data analysis, as well as possible improvements.

\section{The GW background}

The spectrum of the gravitational stochastic background is characterized by the dimensionless density parameter (or closure density) [14, 15]:

$$
\Omega_{g w}\left(\nu_{o}\right)=\frac{1}{\rho_{c}} \frac{d \rho_{g w}}{d \ln \nu_{o}}
$$

where $\rho_{g w}$ is the gravitational energy density, $\nu_{o}$ the frequency in the observer

frame and $\rho_{c}=\frac{3 H_{0}^{2}}{8 \pi G}$ the critical energy density needed to close the Universe today. Throughout this paper, we assume a flat Einstein de Sitter 737 cosmology, with energy density of matter $\Omega_{m}=0.3$, energy density of vacuum $\Omega_{\Lambda}=0.7$ and Hubble parameter $H_{0}=70 \mathrm{~km} \mathrm{~s}^{-1} \mathrm{Mpc}^{-1}$ [16], corresponding to the so-called concordant model derived from observations of distant type Ia supernovae [17] and the power spectra of the cosmic microwave background fluctuations [18].

For a stochastic background of astrophysical origin [20]:

$$
\Omega_{g w}=\frac{1}{\rho_{c} c^{3}} \nu_{o} F_{\nu_{o}}
$$

where the integrated flux (in erg $\mathrm{cm}^{2} \mathrm{~Hz}^{-1} \mathrm{~s}^{-1}$ ) at the observed frequency $\nu_{o}$ is defined as:

$$
F_{\nu_{o}}=\int f_{\nu_{o}} \frac{d R^{o}}{d z} d z
$$

The spectral properties of a single source located at redshift $z$ are given by the fluence (in erg $\mathrm{cm}^{2} \mathrm{~Hz}^{-1}$ ) [19, 20]:

$$
f_{\nu_{o}}=\frac{1}{4 \pi d_{L}^{2}} \frac{d E_{g w}}{d \nu_{o}}=\frac{1}{4 \pi d_{L}^{2}} \frac{d E_{g w}}{d \nu}(1+z)
$$

where $d_{L}=r(1+z)$ is the luminosity distance, $r$ the proper distance, which depends on the adopted cosmological model, and $\nu=\nu_{o}(1+z)$ the frequency in the source frame. In the quadrupolar approximation, the spectral GW energy emitted by a binary system, which inspirals in a circular orbit is given by [21, 22]:

$$
d E_{g w} / d \nu=K \nu^{-1 / 3}
$$


where

$$
K=\frac{(G \pi)^{2 / 3}}{3} \frac{m_{1} m_{2}}{\left(m_{1}+m_{2}\right)^{1 / 3}}
$$

For double neutron stars with masses $m_{1}=m_{2}=1.4 \mathrm{M}_{\odot}$, one obtains $K=5.2 \times 10^{50}$ $\operatorname{erg} \mathrm{Hz}^{-2 / 3}$ and the gravitational frequency at the last stable orbit is assumed to be $\nu_{L S O}=1.5 \mathrm{kHz}[23]$.

The merging of two neutron stars (NSs) occurs long after the formation of the progenitors and this delay must be taken into account when calculating the event rate. The progenitor formation rate per comoving volume is given on the time scale of the observer by:

$$
R_{f}^{o}\left(z_{f}\right)=\lambda \frac{R_{*}\left(z_{f}\right)}{1+z_{f}}
$$

where $R_{*}\left(z_{f}\right)$ is the cosmic star formation rate per comoving volume (SFR) at the time of formation $z_{f}$ and is expressed in $\mathrm{M}_{\odot} \mathrm{Mpc}^{-3} \mathrm{yr}^{-1}$. In our calculations, we consider the recent model of [24], constrained by the Super Kamiokande limit on the electron antineutrino flux from past core-collapse supernovas up to $z_{\max }=6$. The $(1+z)$ term in the denominator corrects the cosmic star formation rate by the time dilatation due to the cosmic expansion. The factor $\lambda$ is the mass fraction converted into the progenitors, assumed to be the same at all redshifts, and given by [2] as the product $\lambda=\beta_{N S} f_{b} \lambda_{N S}$, where $\beta_{N S}$ is the fraction of binaries which remains bounded after the second supernova event, $f_{b}$ the fraction of massive binaries formed among all stars and $\lambda_{N S}$ the mass fraction of NS progenitors derived from a modified Salpeter A IMF with minimal and maximal initial masses of $8 \mathrm{M}_{\odot}$ and $40 \mathrm{M}_{\odot}$, as suggested by [24]. Numerically one obtains $\lambda=3 \times 10^{-5} \mathrm{M}_{\odot}^{-1}$.

The coalescence rate per comoving volume on the time scale of the observer results from the convolution between $R_{f}^{o}$ and the probability distribution of the coalescence time $\tau_{c}$ (or the delay between the formation of the DNS after the second supernova explosion and the coalescence), which depends on the orbital parameters (separation and eccentricity) and neutron star masses, at the time the DNS is formed, and is given by [2] as:

$$
P_{\tau_{c}}\left(\tau_{c}\right)=\frac{0.087}{\tau_{c}} \text { with } \tau_{c} \in[0.2 \mathrm{Myr}-20 \mathrm{Gyr}]
$$

In terms of the cosmic (lookback) time:

$$
T_{c}=T\left(z_{c}\right)=\int_{0}^{z_{c}} \frac{1}{H_{0}} \frac{d z}{E(z)(1+z)}
$$

where

$$
E(\Omega, z)=\sqrt{\Omega_{\Lambda}+\Omega_{m}(1+z)^{3}}
$$

and where $z_{c}$ is the redshift of coalescence, it writes:

$$
R_{c}^{o}\left(z_{c}\right)=R_{c}^{o}\left(T_{c}\right)=\int R_{f}^{o}\left(T_{c}+\tau_{b}+\tau_{c}\right) P_{\tau_{c}}\left(\tau_{c}\right) d \tau_{c}
$$




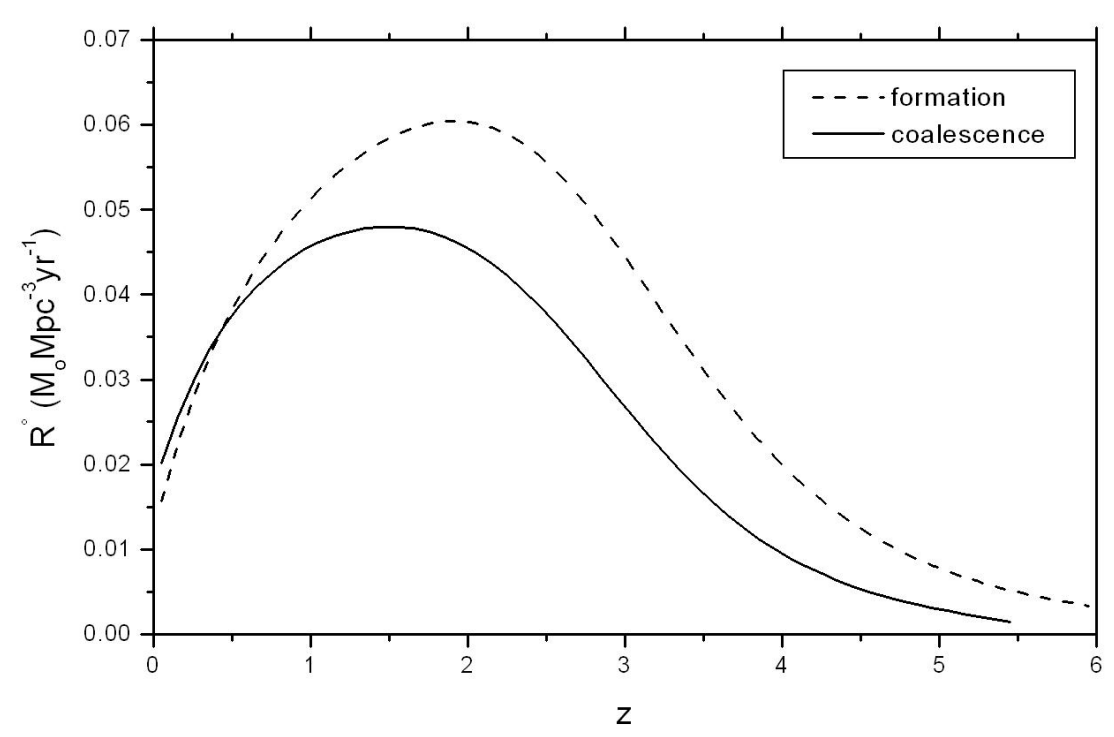

Figure 1. comparison between the formation rate of DNS progenitors and the coalescence rate.

where $\tau_{b}\left(\sim 10^{8} \mathrm{yr}\right)$ is the mean lifetime of the progenitors (or the time for the two massive stars to evolve into two neutron stars) [2]. The lower limit of the integral corresponds to the minimal coalescence time $\tau_{o}=0.2 \mathrm{Myr}$ [2], while the upper limit is fixed by the maximal redshift of the adopted star formation rate and is given by the maximal formation time delayed according to the time to reach the coalescence, namely $T\left(z_{\max }\right)-\tau_{b}-T_{c}$. For $z_{\max }=6, T\left(z_{\max }\right)=T_{\max }=12.5$ Gy.

As one can see in Fig. 1, the coalescence rate is shifted toward lower redshifts, with respect to the formation rate, reflecting the time delay between the formation of the progenitors and the coalescence event.

The event rate per redshift interval in eq. 3, is thus given by multiplying $R_{c}^{o}(z)$ by the element of comoving volume:

$$
\frac{d R^{o}}{d z}=R_{c}^{o} \frac{d V}{d z}
$$

where

$$
\frac{d V}{d z}=4 \pi r^{2} \frac{c}{H_{0}} \frac{1}{E(\Omega, z)}
$$

Combining the expressions above and replacing the constants by their usual values, one obtains:

$$
\Omega_{g w}=8.6 \times 10^{-10} \nu_{o}^{2 / 3} \int_{0}^{z_{\text {sup }}} \frac{R_{c}^{o}(z)}{(1+z)^{4 / 3} E(z)} d z
$$

The upper limit of the integral, which depends on both the maximal emission frequency in the source frame $\nu_{m} a x$ and the maximal redshift of the model of star 
formation history $\left(z_{\max } \sim 6\right)$, is given by:

$$
z_{\text {sup }}= \begin{cases}z_{\max } & \text { if } \nu_{o}<\frac{\nu_{\max }}{1+z_{\max }} \\ \frac{\nu_{\max }}{\nu_{o}}-1 & \text { otherwise }\end{cases}
$$

Consequently, the shape of the spectrum is characterized by a cutoff at the maximal emission frequency and a maximum at a frequency which depends on the shape of both the SFR and the spectral energy density. Before the maximum, $\Omega_{g w}$ increases as $\nu_{o}^{2 / 3}$ (eq. 14).

Besides the spectral properties, it is important to study the nature of the background [25. In the case of burst sources the integrated signal received at $z=0$ from sources up to redshift $z$, would show very different statistical behaviour whether the duty cycle [15]:

$$
D(z)=\int_{0}^{z} \bar{\tau}\left(1+z^{\prime}\right) \frac{d R^{o}}{d z}\left(z^{\prime}\right) d z^{\prime}
$$

defined as the ratio, in the observer frame, of the typical duration of a single event $\bar{\tau}$, to the average time interval between successive events, is smaller or larger than unity. When the number of sources is large enough for the time interval between events to be small compared to the duration of a single event $(D>>1)$, the waveforms overlap to produce a continuous background. Due to the central limit theorem, such backgrounds obey the Gaussian statistic and are completely determined by their spectral properties. They could be detected by data analysis methods in the frequency domain such as the cross correlation statistic presented in the next section 26. On the other hand, when the number of sources is small enough for the time interval between events to be long compared to the duration of a single event $(D<<1)$, the sources are resolved and may be detected by data analysis techniques in the time domain (or the time frequency domain) such as match filtering [27, 28. An interesting intermediate case arises when the time interval between events is of the same order of the duration of a single event. These signals, which sound like crackling popcorn, are known as "popcorn noise". The waveforms may overlap but the statistic is not Gaussian anymore so that the amplitude on the detector at a given time is unpredictable. For such signals, data analysis strategies remain to be investigated [29], since the time dependence is important and data analysis techniques in the frequency domain, such as the cross correlation statistic, are not adapted. The critical redshifts at which the background becomes continuous, popcorn or shot noise will be fixed by the conditions $D\left(z_{c}\right)=1,0.1$ or 0.01 [25].

In our calculations, we considered the last $\sim 1000 \mathrm{~s}$ before the last stable orbit, when more than $96 \%$ of the gravitational energy is released and when the signal range between $10-1500 \mathrm{~Hz}$, in the frequency domain of ground based detectors [12]. At that time, the system has been circularized through GW emission (eq. 5) and all the emission is assumed to take place at the redshift of coalescence. We show that sources at redshifts $z>0.5$ contribute to a truly continuous stochastic background, while sources at redshifts $0.2<z<0.5$ are responsible for a popcorn noise, with duty cycle of 1 and 0.1 respectively (Fig. 3 ). The closure density reaches a maximum of $\Omega_{g w} \sim 7.3 \times 10^{-10}$ 


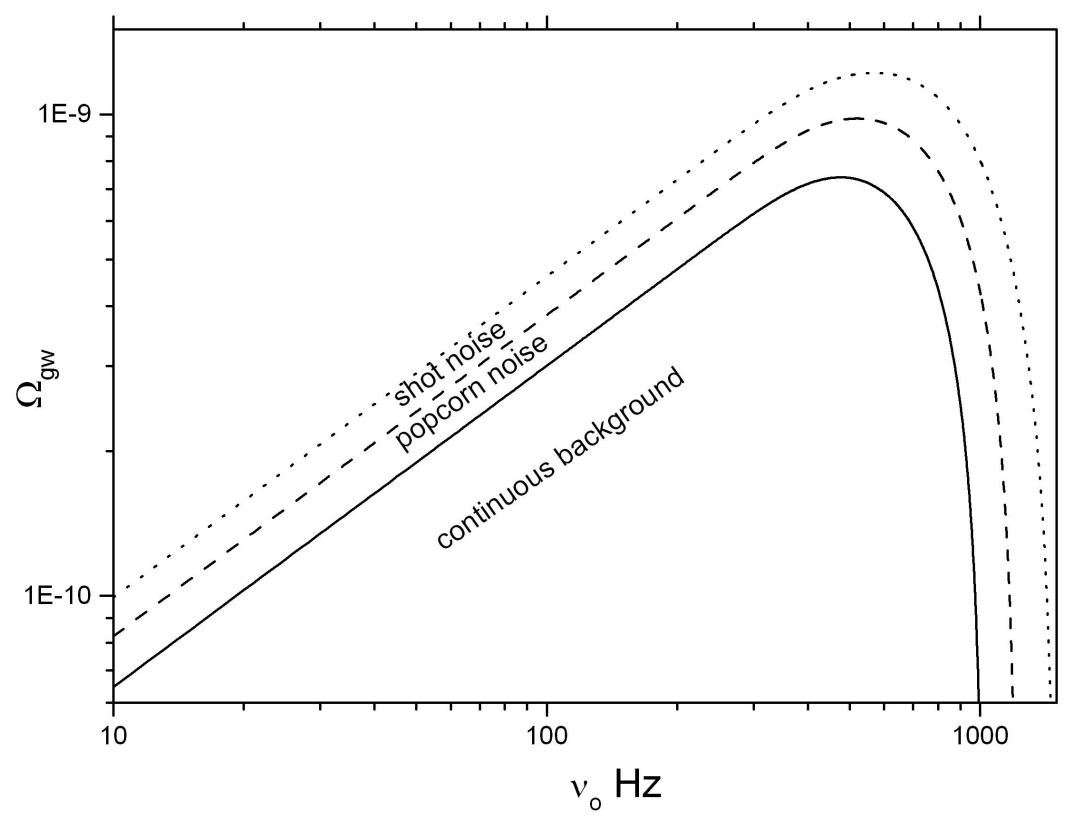

Figure 2. closure density of the continuous background produced by DNS coalescences at $z>0.5$ (continuous line) and of the popcorn contribution corresponding to sources between $z=0.2-0.5$ (dashed line). The signal from the whole population down to $z=0$ is also plotted for comparison (dotted line).

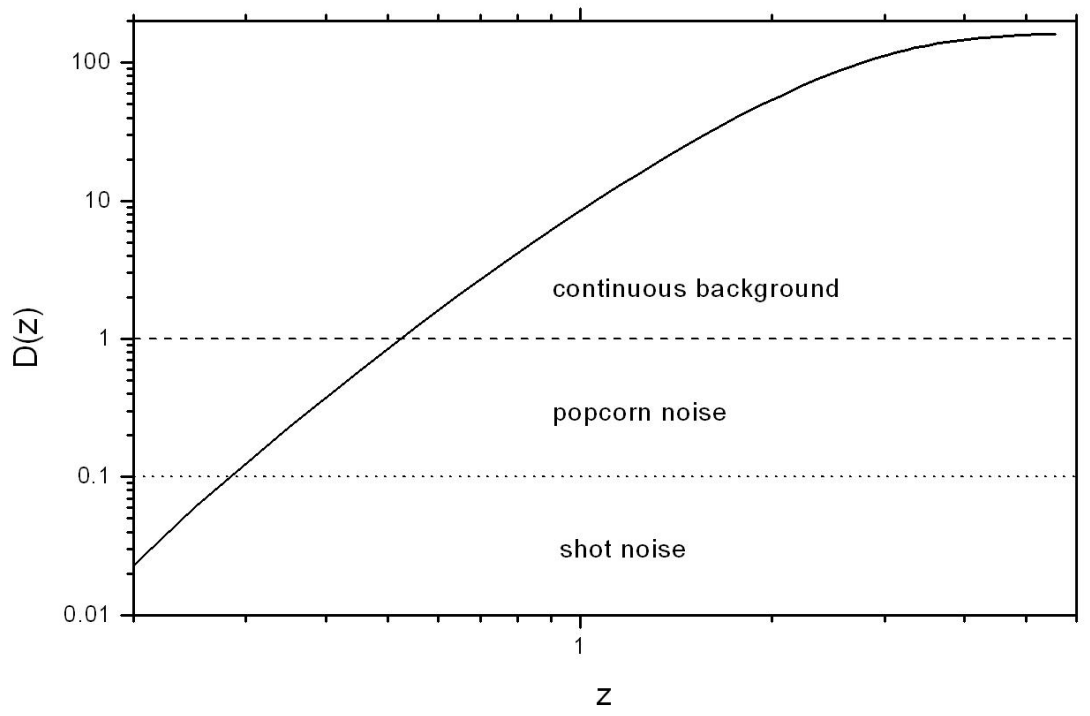

Figure 3. duty cycle from sources up to redshift $z$. The horizontal lines at $D=1$ and $D=0.1$ show the limit of the continuous and the popcorn backgrounds. 
around $480 \mathrm{~Hz}$ for the continuous contribution and of $\Omega_{g w} \sim 9.7 \times 10^{-10}$ around 520 $\mathrm{Hz}$ for the popcorn background (Fig. 2). The total background, including the nearest sources down to $z \sim 0$ is slightly higher, with a maximum of $\Omega_{g w} \sim 1.2 \times 10^{-9}$ at $560 \mathrm{~Hz}$. [9] used a similar procedure to calculate the background in the frequency range of LISA. At lower frequencies our results are comparable (with $\Omega_{g w} \sim 4-5 \times 10^{-10}$ at around $100 \mathrm{~Hz}$ for the total background) besides different assumptions about the SFR, the mass range for NS progenitors and the distribution of the coalescence time. But in [9] the maximum occurs at lower frequencies $(\sim 100 \mathrm{~Hz})$, since being interested in the signal in the range between $10 \mu \mathrm{Hz}$ and $1 \mathrm{~Hz}$, they have set the value of the maximum frequency

to that expected at a separation three times the last stable orbit $\left(\nu_{\max }=0.19 \nu_{L S O}\right)$. Those authors have stressed that their calculations are expected to be accurate in the frequency band of LISA, thus a direct comparison with our predictions for the frequency band of ground based detectors is probably not very meaningful.

\section{Detection}

The optimal strategy to search for a gaussian (or continuous) stochastic background, which can be confounded with the intrinsic noise background of the instrument, is to cross correlate the measurements $s_{i}$ of multiple detectors. When the background is assumed to be isotropic, unpolarized and stationary, the cross correlation product is given by [26]:

$$
Y=\int_{-\infty}^{\infty}{\tilde{s_{1}}}^{*}(f) \tilde{Q}(f) \tilde{s_{2}}(f) d f
$$

where

$$
\tilde{Q}(f) \propto \frac{\Gamma(f) \Omega_{\mathrm{gw}}(f)}{f^{3} P_{1}(f) P_{2}(f)}
$$

is a filter that maximizes the signal to noise ratio $(S / R)$. In the above equation, $P_{1}(f)$ and $P_{2}(f)$ are the power spectral noise densities of the two detectors and $\Gamma$ is the non-normalized overlap reduction function, characterizing the loss of sensitivity due to the separation and the relative orientation of the detectors (see Fig. 4). The optimized $S / N$ ratio for an integration time $T$ is given by [26]:

$$
\left(\frac{S}{N}\right)^{2}=\frac{9 H_{0}^{4}}{16 \pi^{4}} T \int_{0}^{\infty} d f \frac{\Gamma^{2}(f) \Omega_{\mathrm{gw}}^{2}(f)}{f^{6} P_{1}(f) P_{2}(f)}\left(\operatorname{erfc}^{-1}(2 \beta)-\operatorname{erfc}^{-1}(2 \alpha)\right)^{-2} .
$$

The $S / N$ for the main terrestrial interferometer pairs, at design sensitivity and in their advanced configuration, after one year of integration, are given in Table 1, for a detection rate $\alpha=90 \%$ and a false alarm rate $\beta=10 \%$. Expressions for the power spectral densities of actual detectors can be found in [31] (see Fig. 5).

The continuous signal is below the sensitivity that can be obtained by crosscorrelating actual pairs of detectors [26]. For example, considering co-located and coaligned interferometers, such as Virgo or LIGO, we find a maximum signal-to-noise 


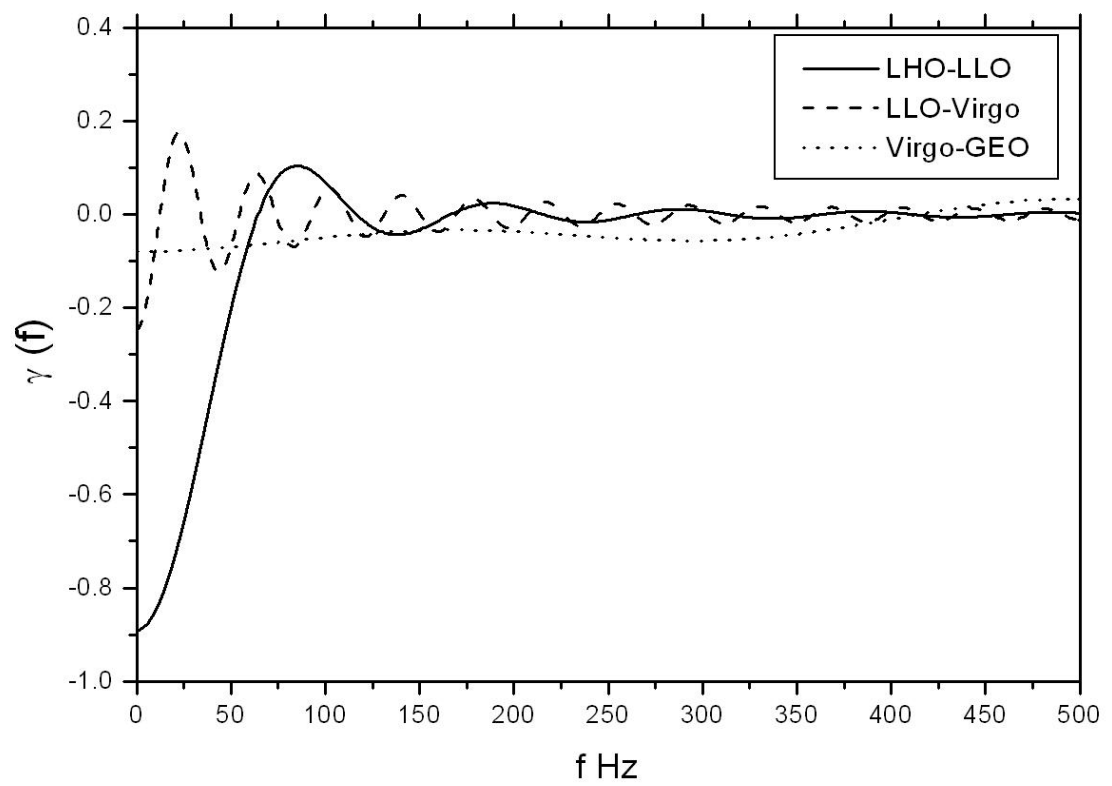

Figure 4. overlap reduction function for the most promising detector pairs. LHO and LLO stand for LIGO Hanford Observatory and LIGO Livingston Observatory.

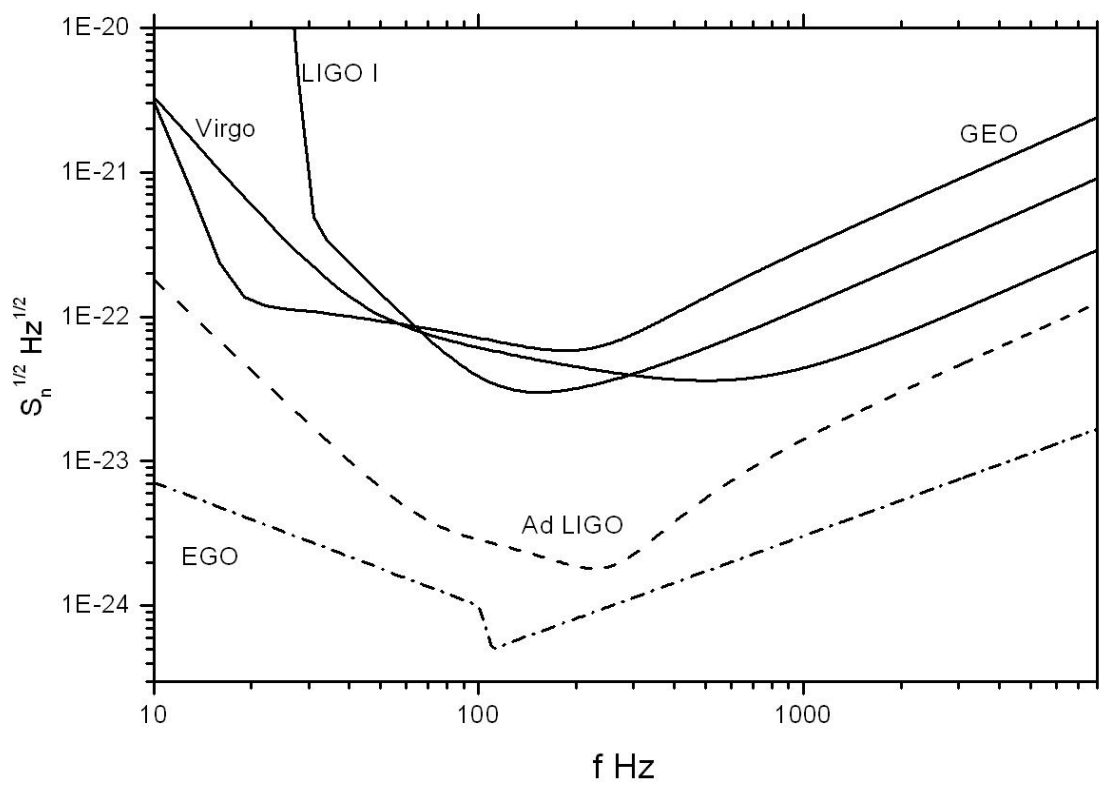

Figure 5. designed sensitivities of the main first generation interferometers (continuous), compared to the planned sensitivities of the advanced interferometer LIGO Ad and the third generation interferometer EGO. 


\begin{tabular}{lccccc}
\hline & LHO-LHO & LHO-LLO & LLO-VIRGO & VIRGO-GEO & EGO-EGO \\
\hline initial & $2.8 \times 10^{-3}$ & $5.5 \times 10^{-6}$ & $6.1 \times 10^{-6}$ & $4.9 \times 10^{-6}$ & - \\
advanced & 0.52 & 0.029 & - & - & - \\
$3^{r} d$ generation & - & - & - & - & 8.4 \\
\hline
\end{tabular}

Table 1. Expected signal-to-noise ratio, corresponding to the continuous background $(D>1)$ and for the actual and future terrestrial interferometer pairs for an integration time $\mathrm{T}=1$ year, a detection rate $\alpha=90 \%$ and a false alarm rate $\beta=10 \%$. LHO and LLO stand for LIGO Hanford Observatory and LIGO Livingston Observatory.

ratio of $S / N \sim 0.003(S / N \sim 0.5)$ for the initial (advanced) configuration. However, the sensitivity of the future third generation of detectors such as EGO, presently in discussion, could be high enough to gain one order of magnitude in the expected signal to noise ratio $(S / N \sim 8)$. On the other hand, the popcorn noise contribution could be detected by new data analysis techniques currently under investigation, such as the maximum likelihood statistic [29], or methods based on the Probability Event Horizon concept [30], which describes the evolution, as a function of the observation time, of the cumulated signal throughout the Universe.

\section{Simulations of the DNS population}

In this section, we introduce Monte Carlo simulations of the extra-galactic population of DNSs and calculate the resulting stochastic background. This method can be extended to any kind of sources, in particular to GW events that are delayed with respect to the formation of the progenitors. The simulations follow the evolution of the system from the birth of the progenitors to the merging of the two neutron stars, after the redshift of formation and the coalescence time have been selected. The difference with the previous simulations of [12, 13], in addition to the update of the initial mass function, the star formation rate and the cosmological model, is that the normalization (the ratio between the real number of DNSs and the number of simulated DNSs or runs) is done in a more realistic way by considering comoving volume elements instead of redshift intervals when following the evolution of the progenitors.

To simulate a population of coalescences observed today in an element of comoving volume, we proceed as follow (see Fig. 6):

(i) The time of formation of the progenitors is selected from the probability distribution

$$
P_{f}\left(T_{f}\right)=\frac{R_{f}^{o}\left(T_{f}\right)}{\int_{0}^{T_{\max }} R_{f}^{o}\left(T_{f}\right) d T_{f}}
$$

defined by normalizing in the interval $0-12.5$ Myr the formation rate of the progenitors (eq. 7),

(ii) The cosmic time $T_{b}$ at which the progenitors have evolved into two neutron stars 


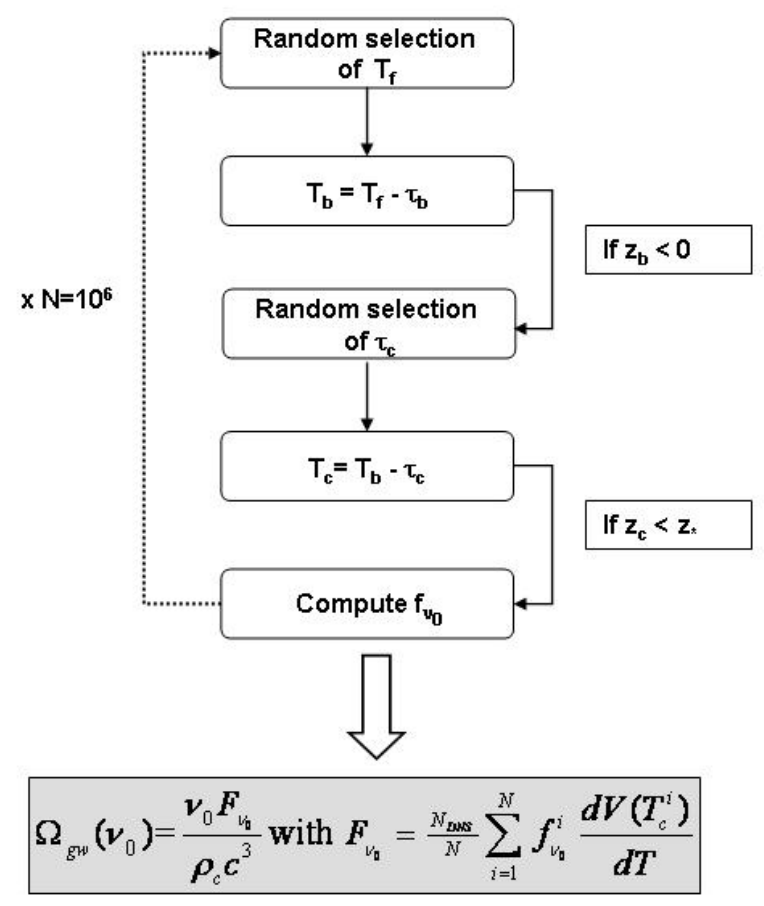

Figure 6. flowchart of the Monte-Carlo simulations described in section 3. Each system is generated with a cosmic time of formation $T_{f}$, (from which the cosmic time of formation of the DNS $T_{b}$ is calculated), and a coalescence time $\tau_{c}$, which defines the coalecence cosmic time $T_{c}$. Only DNSs which coalesce at redshifts $z_{c}<z_{*}$ contribute to the integrated signal; their fluences are calculated and combined with adequate normalization factors (see text) to compute the total flux and the density parameter $\Omega_{g w}\left(\nu_{o}\right)$. The critical redshift to have a continuous stochastic background is $z_{*}=0.4$ $\left(z_{*}=0.2\right.$ for the popcorn noise).

and start to coalesce is given by

$$
T_{b}=T_{f}-\tau_{b}
$$

where $\tau_{b}\left(\sim 10^{8} \mathrm{yr}\right)$ has been defined in the previous section as the mean lifetime of the progenitors.

(iii) The coalescence timescale which depends on both the orbital parameters and the masses of the two neutron stars, is selected from the probability distribution eq. 8 , between 0.2 Myr and 20 Gyr.

(iv) The cosmic time $T_{c}$ at which the coalescence occurs is given by

$$
T_{c}=T_{b}-\tau_{c}
$$


and the corresponding coalescence redshift $z_{c}$ is derived by solving the equation:

$$
T_{c}=\int_{0}^{z_{c}} \frac{d z}{H_{0}(1+z) E(z)}
$$

(v) Each DNS, thus generated, is then sorted into bins of cosmic time $\left[T_{c}^{j} ; T_{c}^{j}+\Delta T_{c}\right]$, for which we calculate the total flux as the sum of all the individual fluences, normalized by the ratio between the total formation rate of the progenitors in the range $0-12.5 \operatorname{Gyr}\left(N_{p}^{o}\right)$ and the number of simulated DNSs $\left(N_{\text {sim }}\right)$ :

$$
F_{j}\left(T_{c}^{j}, \nu_{o}\right)=\frac{N_{p}^{o}}{N_{\text {sim }}} \sum_{i} f\left(T_{c}^{i}, \nu_{o}\right)
$$

with $T_{c}^{i}$ in the range $\left[T_{c}^{j} ; T_{c}^{j}+\Delta T_{c}\right]$ and where

$$
N_{p}^{o}=\int_{0}^{T_{\max }} R_{f}^{o}\left(T_{f}\right) d T_{f}
$$

(vi) The model of the star formation rate being isotropic, the element of comoving volume at cosmic time $T$ is considered as representative of the entire population in the shell between $[T ; T+d T]$. Therefore, the total flux from sources located between $\left[T_{c}^{j} ; T_{c}^{j}+\Delta T_{c}\right]$ writes:

$$
F\left(\nu_{o}\right)=\sum_{j} F_{j}\left(T_{c}^{j}, \nu_{o}\right) \frac{d V}{d T}\left(T_{c}^{j}\right)
$$

or equivalently

$$
F\left(\nu_{o}\right)=\frac{N_{p}^{o}}{N_{\text {sim }}} \sum_{i} f\left(T_{c}^{i}, \nu_{o}\right) \frac{d V}{d T}\left(T_{c}^{i}\right)
$$

The closure density (eq. 2) corresponding to the continuous background $\left(z_{c}>0.5\right)$ is plotted in figure (Fig. 7) and compared with the results obtained in section 1. For a number of runs $N_{\text {sim }}=10^{6}$, the agreement is better than $99.5 \%$, which is accurate enough to validate the Monte Carlo procedure.

\section{Conclusions}

We presented Monte Carlo numerical simulations of the extra-galactic population of double neutron stars and investigated its contribution to the gravitational wave stochastic background. The stochastic background formed by the final stage of the coalescence in the frequency band $10-1500 \mathrm{~Hz}$ is continuous for sources beyond $z \sim 0.5$ and rather a popcorn noise between $0.2<z<0.5$. The closure density of the continuous contribution reaches a maximum of $\Omega_{g w} \sim 7.3 \times 10^{-10}$ at around 480 $\mathrm{Hz}$, which is below the sensitivity of actual and advanced interferometers but may be detectable with the third generation. The popcorn contribution seems more promising with $\Omega_{g w} \sim 9.7 \times 10^{-10}$ at $520 \mathrm{~Hz}$. The advantage of the Monte Carlo simulations, compared to the direct calculation are numerous. On the one hand they permit to study the statistical properties of the background in the time domain, in particular the non gaussian popcorn contribution, for which adapted detection strategies are currently 


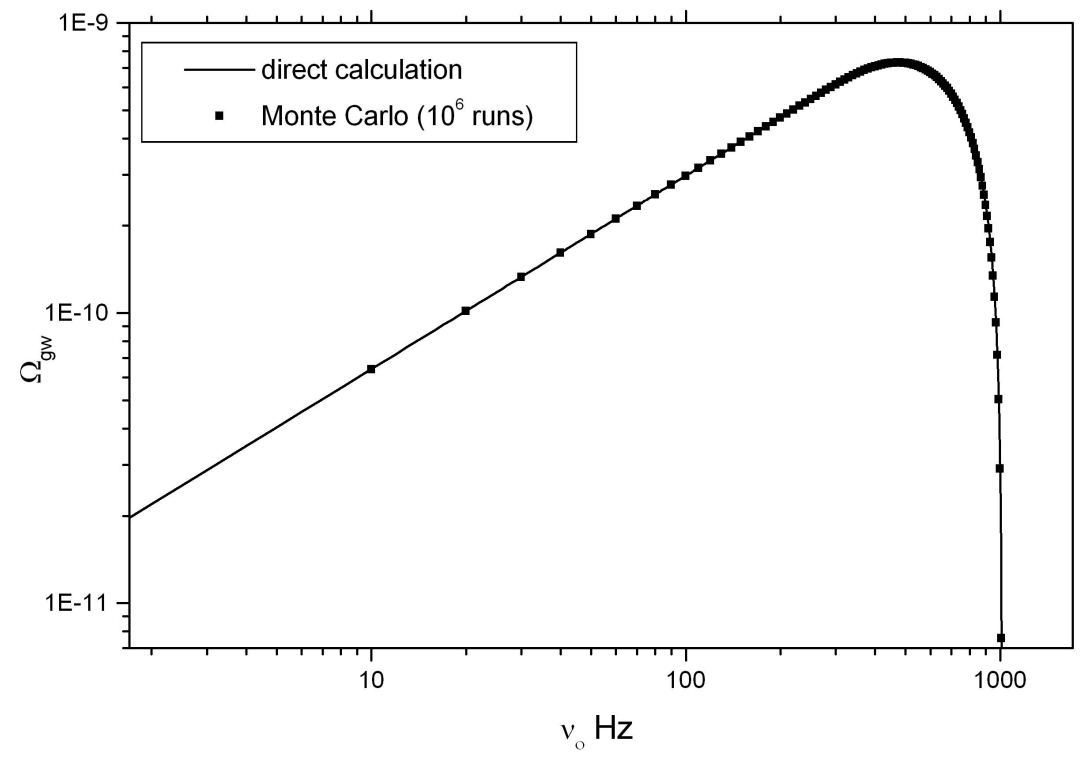

Figure 7. closure density of the continuous background produced by DNS coalescences at $z>0.5$ derived from the direct calculations described in section 1 (continuous line) and from the Monte Carlo simulations described in section 2 (square), for a number of runs $N_{\text {sim }}=10^{6}$. The agreement between the two is better than $99.5 \%$.

under development. The simulation of GW time series [32, 33] that can be injected in the output of a pair of detectors [34, 35], is essential to test and validate data analysis pipelines. On the other hand incorporating new parameters (such as the eccentricity, the orbital separation and the masses of the two stars) can be done in a very simple way. In this work all the initial informations are included in the coalescence time. However to investigate the stochastic background formed by the low frequency inspiral phase, in the frequency domain of the spatial detector LISA, when the system can be highly eccentric [36, 37] emitting GW at higher harmonics to the keplerian frequency [21, 38, 8], one needs to follow the combined evolution of the frequency, the eccentricity and the redshift of emission. This work is currently in progress and will be reported in a future paper.

[1] Regimbau T. de Freitas Pacheco J. A. Spallicci A. and Vincent S. 2005 CQG 22935

[2] de Freitas Pacheco J.A. Regimbau T. Spallici A. and Vincent S. 2006 IJMPD, 15, 235

[3] Evans C.R. Iben I. and Smarr L. 1987 ApJ 323129

[4] Hils D., Bender P.L. and Webbink R.F. 1990 ApJ 36075

[5] Bender P.L. and Hils D. 1997 CQG 141439

[6] Postnov K.A. and Prokhorov M.E. 1998 ApJ 494674

[7] Neleman G., Yungelson L. and S.F. Potergies Zwart S.F. 2001, A $\& A 375890$

[8] Ignatiev V.B. Kuranov A.G. Postnov K.A. and Prokhorov M.E. 2001 MNRAS 327531

[9] Schneider R., Ferrari V., Matarrese S. and Potergies Zwart S.F. 2001 MNRAS 324797

[10] Farmer A.J. and Phinney E.S. 2002 AAS 341225

[11] Cooray A. 2004 MNRAS 35425

[12] Regimbau T. and de Freitas Pacheco J. A. 2006 ApJ 642455 
[13] Regimbau T. 2007 PRD 75043002

[14] Allen B., 1997, in Proc. of Relativistic Gravitation and Gravitational Radiation, ed. Marck J. A. and Lasota J. P. (Cambridge:University Press), 373

[15] Maggiore M. 2000 PhR 331283

[16] Rao S.M. Turnshek D.A. and Nestor D.B. 2006 ApJ 636610

[17] Perlmutter S. et al. 1999 ApJ 517565

[18] Spergel et al. 2003 ApJS 148175

[19] Peacock J.A. 1999 Cosmological Physics. Cambridge University press, Cambridge

[20] Ferrari V. Matarrese S. and Schneider R. 1999 MNRAS 303258

[21] Peters P.C. and Mathews J. 1963 PRD 131435

[22] Misner C.W. Thorne K.S. and Wheeler J.A. 1995 Gravitation 19th edn. Freeman New York

[23] Sathyaprakash B.S. 2002 gr-qc/0012014

[24] Hopkins A.M. Beacom J. 2006 ApJ in press (asto-ph/0601463)

[25] Coward D. and Regimbau T. 2006 New Astronomy Reviews 50461

[26] Allen B. and Romano J. 1999 PRD 5910

[27] Arnaud, N. et al. 1999 PRD 59082002

[28] Pradier T. et al. 2001 PRD 63042002

[29] Drasco S. Flanagan E. 2003 PRD 678

[30] Coward D. and Burman R.R. 2005 MNRAS 361362

[31] Damour T. Iyer B.R. and Sathyaprakash B.S. 2001 PRD 63044023

[32] Coward D. Burman R.R. and Blair D. 2002 MNRAS 329411

[33] Coward D. Lilley M. Howell E. Burman R.R. Blair D. 2005 MNRAS 364807

[34] Bose S. et al. 2003 CQG 20S 677

[35] Cella G. et al. 2007 gr-qc/07042983

[36] Chaurasia H.K. and Bailes M. 2005 ApJ 6321054

[37] Ihm M. Kalogera V. and Belczynski K. 2006 ApJ 652540

[38] Peters P.C. 1964 PRD 1361224 